\title{
Strongly Interacting Matter at Extremely High Energy Density
}

\section{McLerran}

Physics Dept, Bdg. 510A, Brookhaven National Laboratory, Upton, NY-11973, USA

RIKEN BNL Research Ctr, Brookhaven National Laboratory, Upton, NY 11973, USA

Physcs Department, China Central Normal University, Wuhan, China

\begin{abstract}
A variety of new forms of matter have been suggested from studies of Quantum Chromodynamics (QCD). Matter in thermal equilibrium includes the Quark Gluon Plasma which is high temperature matter, Quarkyonic Matter which includes various forms of confined matter with novel realizations of mass generation, or chiral symmetry breaking, and color superconductivity. High energy density gluon matter, the Color Glass Condensate, describes the high density gluon states that control the high energy limit of QCD. In collisions of nuclei, a transient Glasma state might be made that is highly coherent, weakly coupled but strongly interacting. This Glasma ultimately thermalizes into a QGP.
\end{abstract}

International Symposium on on the Quest for the Origin of Particles and the Universe Kobayashi Maskawa Institute

Nagoya, Japan

Dec. 11-13, 2013 


\section{Introduction}

Several very important unresolved issues in contemporary nuclear physics are

- What is the high energy limit for the collisions of strongly interacting particles?

- What are the possible forms of high energy density matter?

- How might such matter be produced and studied?

In recent years a variety of new forms of high energy matter have been inferred from the properties of the theory of strong interactions, QCD. These forms of matter may be produced in such collisions, and are most important at the highest collision energy. An understanding of this matter will provide insight into many aspects of the above questions.

The specific kinds of matter that will be described in more detail in the latter part of this talk are

- Matter in Thermal Equilibrium

1. Hadronic Matter

2. The Quark Gluon Plasma QGP

3. Quarkyonic Matter

4. Color Superconductivity

\section{- Matter Not in Thermal Equilibrium}

1. The Color Glass Condensate (CGC)

2. Glasma

\section{Matter in Thermal Equilibrium}

The various types of matter associated with strong interactions is conceptualized in the temperature baryon number chemical potential plane in Fig. 1[1].

\subsection{Hadronic Matter}

Hadronic matter is the confined world of nucleons and mesons. This corresponds roughly to temperatures $T \leq 200 \mathrm{MeV} \sim \Lambda_{Q C D}$, and baryon chemical potential less than or of the order the nucleon mass. If we were to express this in terms of quark number chemical potentials, this too would be $\mu_{q} \sim \mu_{B} / 3 \leq 300-400 \mathrm{MeV}$. In the confined world, masses are generated by the condensation of an effective scalar field that breaks chiral syemmetry. This condensate $\langle\sigma\rangle$ is spatially homogeneous. In the limit of a large number of colors, the energy density of hadroninc matter is independent of the number of colors, $N_{c}$ 


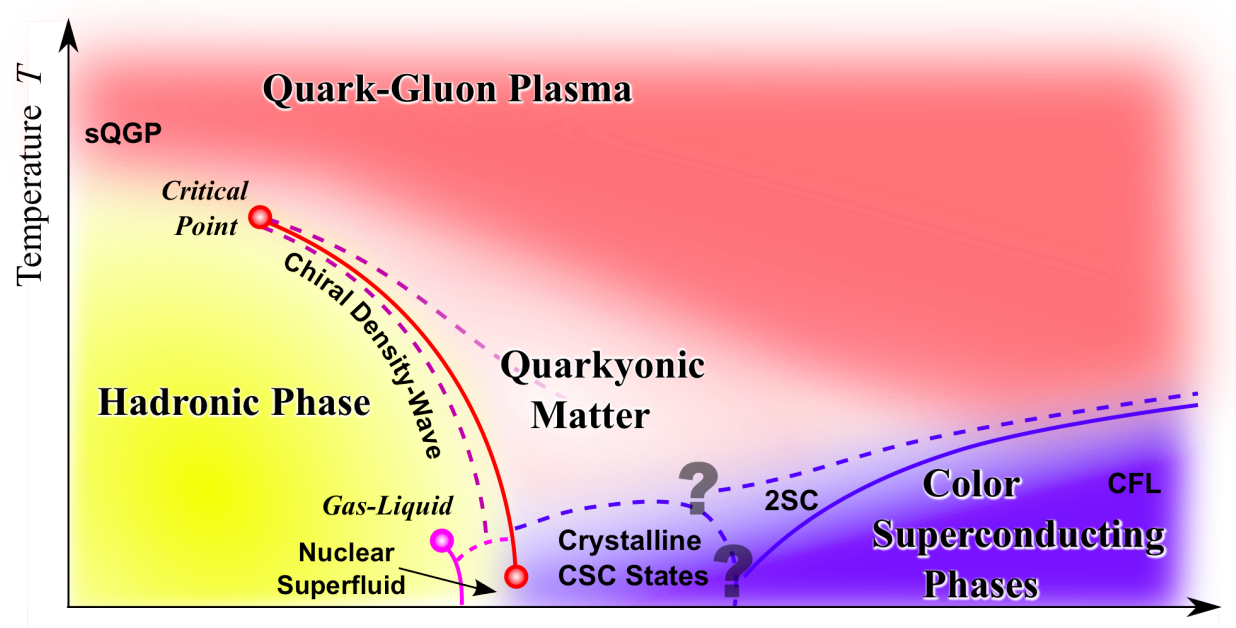

Baryon Chemical Potential $\mu_{\mathrm{B}}$

Figure 1: The QCD Phase Diagram in terms of temperature and baryon number density[1].

\subsection{The Quark Gluon Plasma}

The Quark Gluon Plasma is matter at high temperature $T \geq \Lambda_{Q C D}$ and high baryon chemical potential[2]. In such a high density environment, the quarks and gluons are deconfined. The chiral condensate has been evaporated, and chiral symmetry is restored, so that quark masses may be taken to be small. There is only a cross over between hadronic matter and the QGP at low baryon chemical potential for finite quark masses. There is good lattice Monte-Carlo information about the static properties of the QGP at low baryon number density. At high baryon number density, lattice Monte Carlo methods do no work well because of issues related to the fermionic nature of quarks. In the limit of a large number of colors, the energy density and pressure of the Quark Gluon Plasma scale as $N_{c}^{2}$

\subsection{Quarkyonic Matter}

Quarkyonic matter is best understood in the limit of a large number of colors, $N_{c}$ [3]. In this limit, baryon masses are very large, and the quark-antiquark potential in vacuum is linear at large distances. In Hadronic Matter, there are therefore no baryons, and quark anti-quark pairs and gluons are all bound into mesons. Quarkyonic matter is confined matter but has a sufficiently large chemical potential so that there is a very high density of baryons. In the Fermi sea, the matter can be thought of as quarks, since interactions are at momentum scales large compared to that of the confinement scale. On the Fermi surface however, quarks are all bound into baryons. The excitations of the system corresponding to quark anti-quark pairs are bound mesons and glueballs. Quarkyonic matter has chiral symmetry broken, but it is broken in translationally non-invrariant modes[4]. There are a variety of possible realizations of breaking of chiral symmetry and this might lead to a rich phase structure. Quarkyonic matter is present for temperatures greater than that of the confinement scale but less than $\sqrt{N_{c}} \Lambda_{Q C D}$. On account of the breaking of translational invariance, Quarkyonic matter is isolated from the Hadronic Matter and the Quark Gluon Plasma 
by phase transitions. In the limit of a large number of color, the energy density and pressure of Quarkyonic Matter scale as $N_{c}$

\subsection{Color Superconductivity}

At very low temperatures and high baryon density one expects the formation of condensates of colored Cooper pairs of quarks[5]. This is because in order to make a Cooper pair condensate, one only needs a bound state of a quark pairs. In the scattering of such pairs there is an attractive scattering channel for an antisymmetric in color pairing of the quarks. Near the Fermi surface, the dimensionality of interactions is reduced by the constraint that momenta are close to that of the Fermi surface, and in this reduced dimensional momentum space, an attractive interaction always implies a bound state.

The actual realization of the mode of color superconductivity is quite complicated and the literature is large. It is also remarkable that color superconductivity is a very strong prediction of $\mathrm{QCD}$, and can be shown by weak coupling methods to exist at very high density for some range of temperature.

\subsection{Generic Features of the Phase Diagram: The Triple Point and the Critical End Point}

If Quarkyonic matter exists with broken translational symmetry, then this matter forms an island in the phase diagram surrounded by lines of phase transitions[6]. There is therefore a region where the QGP, Hadronic Matter and Quarkyonic Matter meet. This point is a triple point. If this triple point corresponds to a first order phase transition, then at lower baryon density, this transition will disappear. The point at which such a first order transition disappears is the critical end point. For truly massless quarks, this line might never end, or the triple point might join a line of second order transitions. For finite masses, there may be approximate critical behavior near where the line of second order transitions would have been for massless quarks.

\section{Matter Not in Thermal Equilibrium}

\subsection{The Color Glass Condensate}

The Color Glass Condensate (CGC) is the matter from which gluons form the part of a high energy hadron responsible for multi-particle production[7]. The condensate might be observed from electron-proton scattering experiments, and is a consequence of the increase in the number of gluons in the hadron without bound as the energy increases. If we look at gluons produced near central rapidity and define $x=p_{T} / E_{C M}$, where $p_{T}$ is the produced hadron energy and $E_{C M}$ is the center of mass energy per nucleon of the particles in the beam, the density of gluons appears to grow as $1 / x^{\delta}$ where $\delta \sim .2-.3$. On the other hand, the total tranverse size of the collision region grows slowly with energy, $\pi R^{2} \sim \ln ^{2}\left(E_{C M} / \Lambda_{Q C D}\right)$. The density of gluons therefore increases as energy increases. This means the coupling constant must become small, $\alpha_{S}<<1$.

How can this be true and the matter still be strongly interacting? If the quantum occupancy of gluonic states is $d N / d y d^{2} p_{T} d^{2} x_{T} \sim 1 / \alpha_{s}$, then the typical gluon field strength will be $|A|^{2} \sim 1 / \alpha_{s}$. This large value of the field strength is associated with coherence of the gluon field. The CGC is an example of a system with intrinsic weak coupling but very strong interactions. 
The Coior Glass Condensate derives its name from several observations. The gluons that compose it are colored. The high density of gluons forms dynamically and is at the maximum allowed when coherent gluon interactions become strong, so it is a condensate. It has much in common with the Higgs condensate and Bose condensates found in atomic systems. The word glass arises from technical considerations. The sources that produce a gluon field at small $x$ are generated by fields at a larger values of $x$. These gluons at large values of $x$ (large longitudinal momentum) have their time scales of interaction dilated relative to the natural time scales at small $x$. The small $x$ fields therefore evolve over much longer times than their natural times scales, as do the atoms in an ordinary glass. This results in a statistical matrix approach based in the renormalization group, and the mathematical theory that results is similar to that of spin glasses.

There is a momentum scale associated with the density of particles in the transverse plane, $Q_{s a t}^{2}$, the saturation momentum. At high gluon density, $Q_{s a t}^{2}>>\Lambda_{Q C D}^{2}$, so that $\alpha_{S}\left(Q_{s a t}\right)<<1$.

The gluon fields at high density are simply Lorentz boosted Coulomb fields. They are transverse to the beam direction. They are random in color and polarization. The fluctuations in the density, color and polarization are generated by a weight function determined by the theory of the CGC.

\subsection{Colliding Sheets of Colored Glass}

Because the phase space density of gluons is large, the gluons in the CGC are described by classical fields[8]. We can therefore consider their collision as a problem in solving the time dependent classical field equations. . In Fig. 1, two sheets of CGC are shown on the left hand side of the figure. After the collision, the fields appear as on the right hand side of the figure.
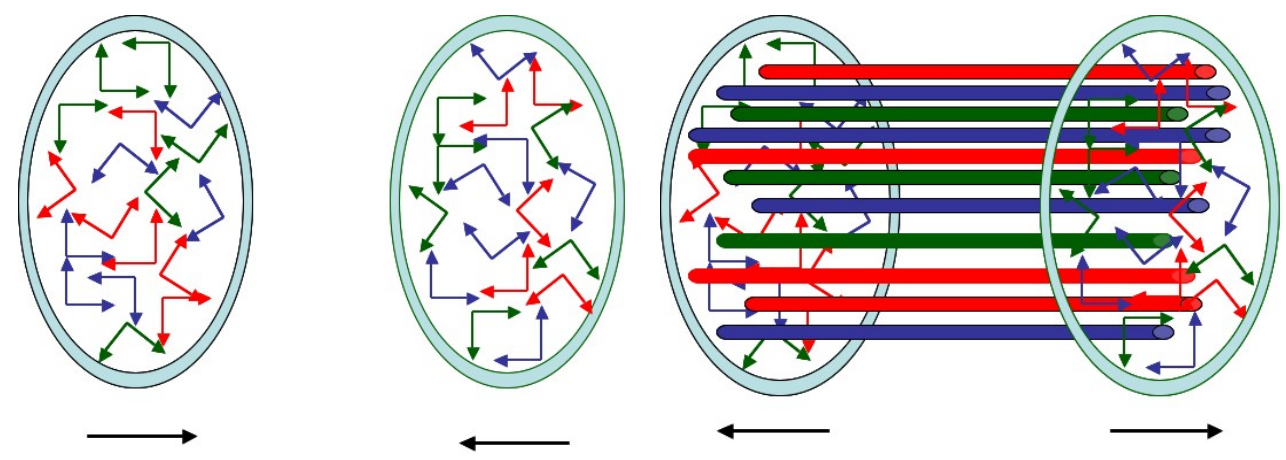

Figure 2: Collision of two sheets of CGC to make a Glasma.

In the collision of two sheets of CGC, longitudinal colored fields are produced. This results because during the collision an equal and opposite color electric and color charge density is deposited on the sheets. The longitudinal lines of color electric and color magnetic fields result because they must connect the charge on one sheet with the opposite charge on the other. Because there are both color electric and color magnetic fields, there is initially a large density of topological charge $\vec{E} \cdot \vec{B}$. Topological charge can induce net helicity since the electric field will accelerate a particle, and the magnetic field induces a direction of spiraling along the direction of motion. The induced helicity is correlated with the sign of the topological charge. 
These fields evolve in time and eventually become dilute. They produce radiation fields which can be interpreted as gluon production. It appears to be a well defined classical problem. This however turns out to be untrue[8]-[16]! In fact these initial conditions present a problem in classical chaos and turbulence. Chaos and turbulence typically results when initial conditions are in some restricted region of phase space. For the Glasma, this is associated with the boost invariance of the initial conditions. In order to thermalize the system, one needs fluctuations in the longitudinal direction of magnitude equal to that in the transverse direction.

If one considers an infinitesimal fluctuation in the longitudinal structure of the field, one finds that it grows exponentially. In this case there are initial seeds of fluctuation in the longitudinal wave function associated with the quantum fluctuations in the initial wave function that describes this system. There is a direct analogy here with computations done in cosmology, where an initial axion or dilation field which is highly coherent must decay into a thermal spectrum[17]. Again, the theory is of a very weakly coupled field, and the evolution of the field appears to be strongly interacting as a result of classical coherence.

There have been attempts to numerically simulate the Glasma, and the results have not yet converged between various groups[18]-[22].

\subsection{Bose Condensation and Topological Excitations}

The initial conditions one starts with are coherent fields that are highly over occupied in a limited region of phase space. The system will naturally diffuse to other regions of phase space as time evolves. To thermalize the system, one will need to evolve into the ultraviolet[23]-[25]. The initial momentum scale $Q_{\text {sat }}$ will split into two evolving scales. The first is an infrared scale $\Lambda_{c o h}$. This scale is where the fields have occupancy of order $1 / \alpha_{S}$. There is also an ultraviolet scale above which the field falls rapidly to zero, $\Lambda_{U V}$. At early times $\Lambda_{U V} \sim \Lambda_{c o h} \sim Q_{s a t}$. Thermalization can occur when they $\Lambda_{U V} \sim T$ and $\Lambda_{c o h} \sim \alpha_{S} T$, that is the scales must separate by an amount $\Lambda_{U V} / \Lambda_{c o h} \sim 1 / \alpha_{S}$. This clearly will take some time, and as the process is diffusive may take times parametrically long compared to the inverse saturation momentum. Even though this may take a long time, one may still get approximate hydrodynamic behavior.

In addition to diffusion to higher momentum scale, we might ask if it is possible to diffuse to lower momentum scales. However, as one evolves into the infrared, the minimum possible momentum is $\vec{p}=0$. If there is a flow to the infrared, one will develop a Bose condensate, that is a large occupation of the zero momentum mode. This occurs in scalar computations. It is not clear what happens in the gauge field case, since it is difficult to introduce a gauge invariant order parameters corresponding to the condensate.

One can address the issue of condensation within the Abelian Higgs model[26]. Here the order parameter is the Higgs field, and it is not gauge invariant. One might be tempted to conclude that there is no non-trivial phase structure in this theory. This is not true. There are three possible phases: A normal phase corresponding to no Higgs condensate. In this phase, there are long range magnetic fields. At very large distances, these fields can be screened by magnetic monopole excitations. There is also a superconducting phase where magnetic fields are confined into flux tubes. Within the superconducting phase there are two possibilities: Vortices corresponding to confined magnetic fields either attract or repel. If they attract, the vortices coalesce into macroscopic regions of condensed vortices. 


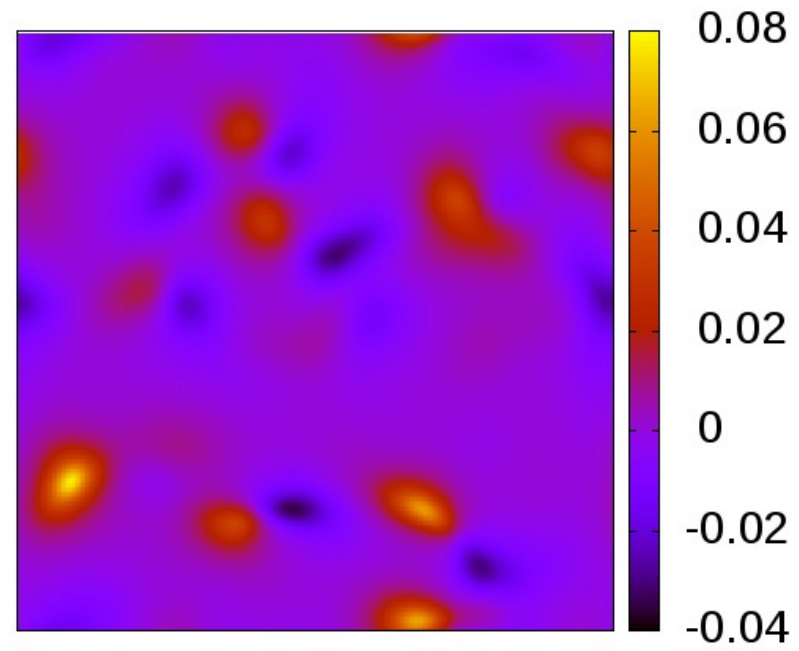

Figure 3: Vortices in the turbulent Abelian Higgs model

If one simulates the evolution of a turbulent Abelian Higgs model, one can indeed, see the effects of the different phases. One can generalize the Higgs field expectation value to a correlation function made gauge invariant by the introduction of Wilson line of electromagnetic field. The phases are characterized by the large separation limit of $\left\langle\phi^{*}(y) e^{i \int_{x}^{y} d x^{\prime} \cdot A\left(x^{\prime}\right)} \phi(x)\right\rangle$. In addition, one can see non-trivial aspects of the condensation in the form of vortices, Fig. 3. In addition, there are entirely non-trivial effects associated with the condensation of charged domains of the Higgs' field, the so called qballs, as shown in Fig. 4. What happens in the turbulent Yang-Mills Glasma is

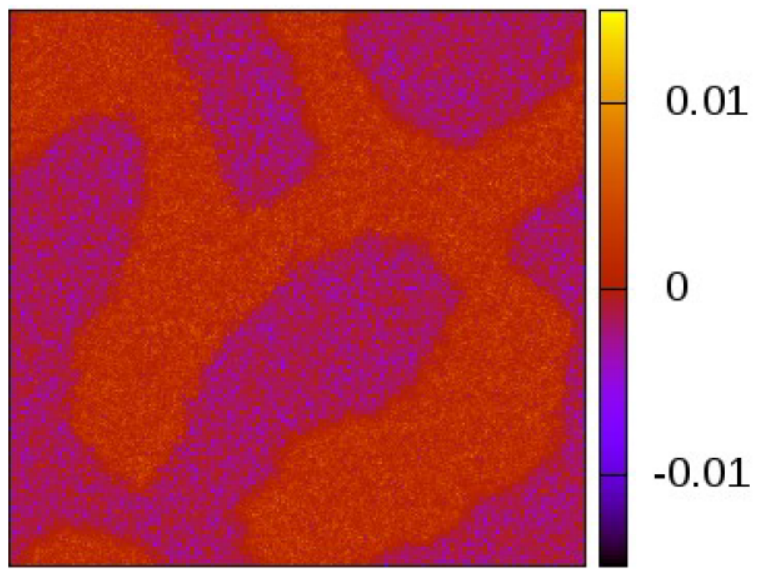

Figure 4: Qballs in the turbulent Abelian Higgs model

not known at present.

Why might we expect such nontrivial phenomena, and why might it be interesting? The tur- 
bulent fields are fluctuating and they are large. They are strongly interacting since they are highly coherent. In the Yang-Mills vacuum, non perturbative phenomena are associated with fluctuations of vacuum fields that interact strongly because the coupling is large. Such non-perturbative phenomena were unexpected when Yang-Mills theories were first introduced. By analogy, we might expect a rich variety of novel unexpected phenomena in the turbulent Glasma,

\section{Hints about New Forms of Matter}

There are a variety of experimental environments in which one can study the properties of high energy density hadronic matter. Thermally equilibrated matter and the Glasma might be produced in heavy ion collisions. The CGC surely play a role in all high energy hadronic collisions and might be precisely studied in high energy electron-hadron collisions. High energy hadron-nucleus collisions are perhaps best suited for these studies because at the same beam energy, the CGC will be at higher density in nuclei than in a proton. High baryon density matter might be found in low to intermediate energy nuclear collision, and might play a role in the high density cores of neutron stars.

\section{Acknowledgements}

The research of L. McLerran is supported under DOE Contract No. DE- AC02-98CH10886.

\section{References}

[1] K. Fukushima and T. Hatsuda, Rept. Prog. Phys. 74 (2011) 014001 [arXiv:1005.4814 [hep-ph]].

[2] G. Boyd, J. Engels, F. Karsch, E. Laermann, C. Legeland, M. Lutgemeier and B. Petersson, Nucl. Phys. B 469 (1996) 419 [hep-lat/9602007].

[3] L. McLerran and R. D. Pisarski, Nucl. Phys. A 796 (2007) 83 [arXiv:0706.2191 [hep-ph]].

[4] T. Kojo, Y. Hidaka, L. McLerran and R. D. Pisarski, Nucl. Phys. A 843 (2010) 37 [arXiv:0912.3800 [hep-ph]].

[5] K. Rajagopal and F. Wilczek, In *Shifman, M. (ed.): At the frontier of particle physics, vol. 3* 2061-2151 [hep-ph/0011333].

[6] A. Andronic, D. Blaschke, P. Braun-Munzinger, J. Cleymans, K. Fukushima, L. D. McLerran, H. Oeschler and R. D. Pisarski et al., Nucl. Phys. A 837 (2010) 65 [arXiv:0911.4806 [hep-ph]].

[7] F. Gelis, E. Iancu, J. Jalilian-Marian and R. Venugopalan, Ann. Rev. Nucl. Part. Sci. 60 (2010) 463 [arXiv:1002.0333 [hep-ph]].

[8] A. Kovner, L. D. McLerran and H. Weigert, Phys. Rev. D 52 (1995) 6231 [hep-ph/9502289].

[9] A. Kovner, L. D. McLerran and H. Weigert, Phys. Rev. D 52 (1995) 3809 [hep-ph/9505320].

[10] A. Krasnitz and R. Venugopalan, Phys. Rev. Lett. 84 (2000) 4309 [hep-ph/9909203].

[11] T. Lappi, Phys. Rev. C 67 (2003) 054903 [hep-ph/0303076].

[12] T. Lappi and L. McLerran, Nucl. Phys. A 772 (2006) 200 [hep-ph/0602189].

[13] S. Mrowczynski, Phys. Lett. B 214 (1988) 587 [Erratum-ibid. B 656 (2007) 273]. 
[14] P. Romatschke and R. Venugopalan, Phys. Rev. Lett. 96 (2006) 062302 [hep-ph/0510121].

[15] P. Romatschke and R. Venugopalan, Phys. Rev. D 74 (2006) 045011 [hep-ph/0605045].

[16] K. Dusling, T. Epelbaum, F. Gelis and R. Venugopalan, Nucl. Phys. A 850 (2011) 69 [arXiv:1009.4363 [hep-ph]].

[17] S. Y. .Khlebnikov and I. I. Tkachev, Phys. Rev. Lett. 77 (1996) 219 [hep-ph/9603378].

[18] J. Berges, K. Boguslavski, S. Schlichting and R. Venugopalan, arXiv:1303.5650 [hep-ph].

[19] J. Berges, K. Boguslavski, S. Schlichting and R. Venugopalan, arXiv:1311.3005 [hep-ph].

[20] T. Epelbaum and F. Gelis, Phys. Rev. Lett. 111 (2013) 232301 [arXiv:1307.2214 [hep-ph], arXiv:1307.2214 [hep-ph]].

[21] T. Epelbaum and F. ï£jo. Gelis, arXiv:1401.1666 [hep-ph].

[22] J. Berges, K. Boguslavski, S. Schlichting and R. Venugopalan, arXiv:1312.5216 [hep-ph].

[23] J. -P. Blaizot, F. Gelis, J. -F. Liao, L. McLerran and R. Venugopalan, Nucl. Phys. A 873 (2012) 68 [arXiv:1107.5296 [hep-ph]].

[24] A. Kurkela and G. D. Moore, JHEP 1112 (2011) 044 [arXiv:1107.5050 [hep-ph]].

[25] J. -P. Blaizot, J. Liao and L. McLerran, Nucl. Phys. A 920 (2013) 58 [arXiv:1305.2119 [hep-ph]].

[26] T. Gasenzer, L. McLerran, J. M. Pawlowski and Dï£¡n. Sexty, arXiv:1307.5301 [hep-ph]. 